Supporting Information

\title{
Developing riboswitch-mediated gene regulatory controls in thermophilic bacteria
}

Supporting Figure S1: Aptamer domain of the purine riboswitches in T. saccharolyticum Supporting Figure S2: Normalized fluorescence of cells expressing super-folder GFP Supporting Figure S3: Growth of G. thermoglucosidasius with and without guanosine added to the media.

Supporting Figure S4. Growth of G. thermoglucosidasius with and without 2-aminopurine added to the media.

Supporting Figure S5: Tsac 2584 non-binding mutant control

Supporting Figure S6: Plasmid map of pJMK06

Supporting Figure S7: Growth comparison between $\Delta h p t$ ("wild-type") and $\Delta a d h E$.

Supporting Table S1: Sequences for riboswitch constructs

Supporting Table S2: Plasmids developed for this study

Supporting Table S3: Quantification of $C$. thermocellum fermentation products

Supporting Methods

Supporting References 


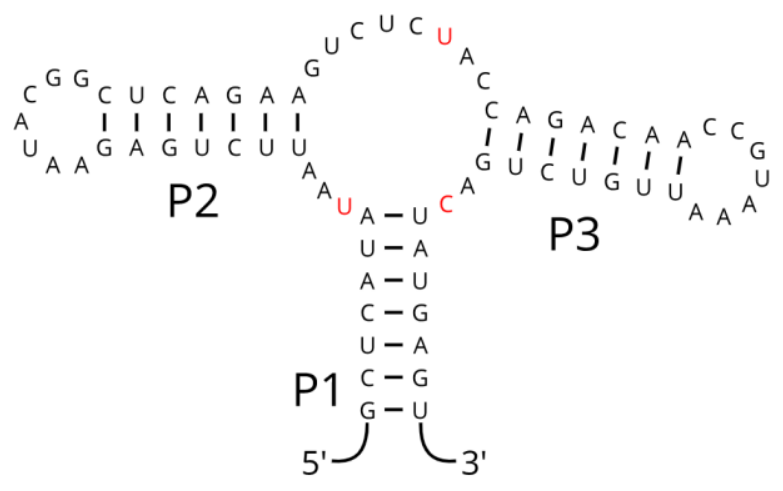

T. saccharolyticum 2584

Purine Riboswitch $\Delta G^{\circ}=-19.25 \mathrm{kcal} / \mathrm{mol}$

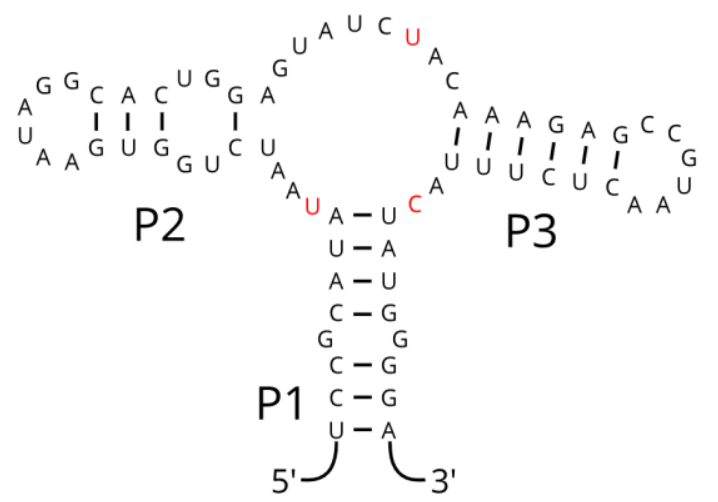

T. saccharolyticum 2588

Purine Riboswitch $\Delta G^{\circ}=-15.95 \mathrm{kcal} / \mathrm{mol}$

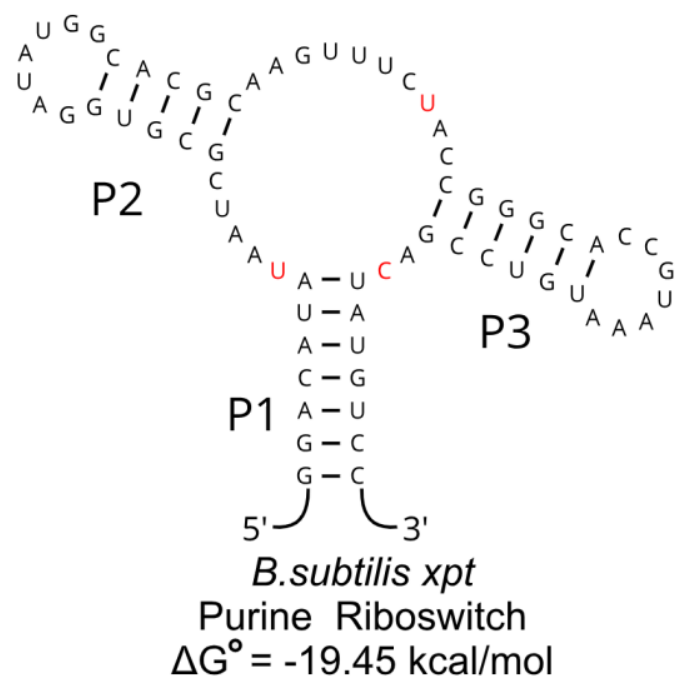

Figure S1. Secondary structure of the purine riboswitch aptamers from $T$. saccharolyticum.

The aptamers for the riboswitch sequences identified in $T$. saccharolyticum contain the canonical structure of the purine riboswitch aptamer which is composed of a three-way junction surrounded by three paired regions P1, P2, and P3. The aptamer domain structure of the $B$. subtilis purine riboswitch is provided for comparison. It can be observed that the aptamer domain of the of the Tsac 2588 riboswitch contains a bulge in the P2 region. This could be related to its differential regulatory activity compared to 
the Tsac 2584 riboswitch. Nucleotides in red dictate the binding specificity of the purine riboswitches. From 5' to 3', if the sequence of these 3 nucleotides is U,U,C the aptamer domain bind specifically guanine. If the sequence is $U, U, U$, then the aptamer domain binds adenine. The folding energies for the secondary structure were calculated using the mFOLD software version 3.6 at $37^{\circ} \mathrm{C}$.

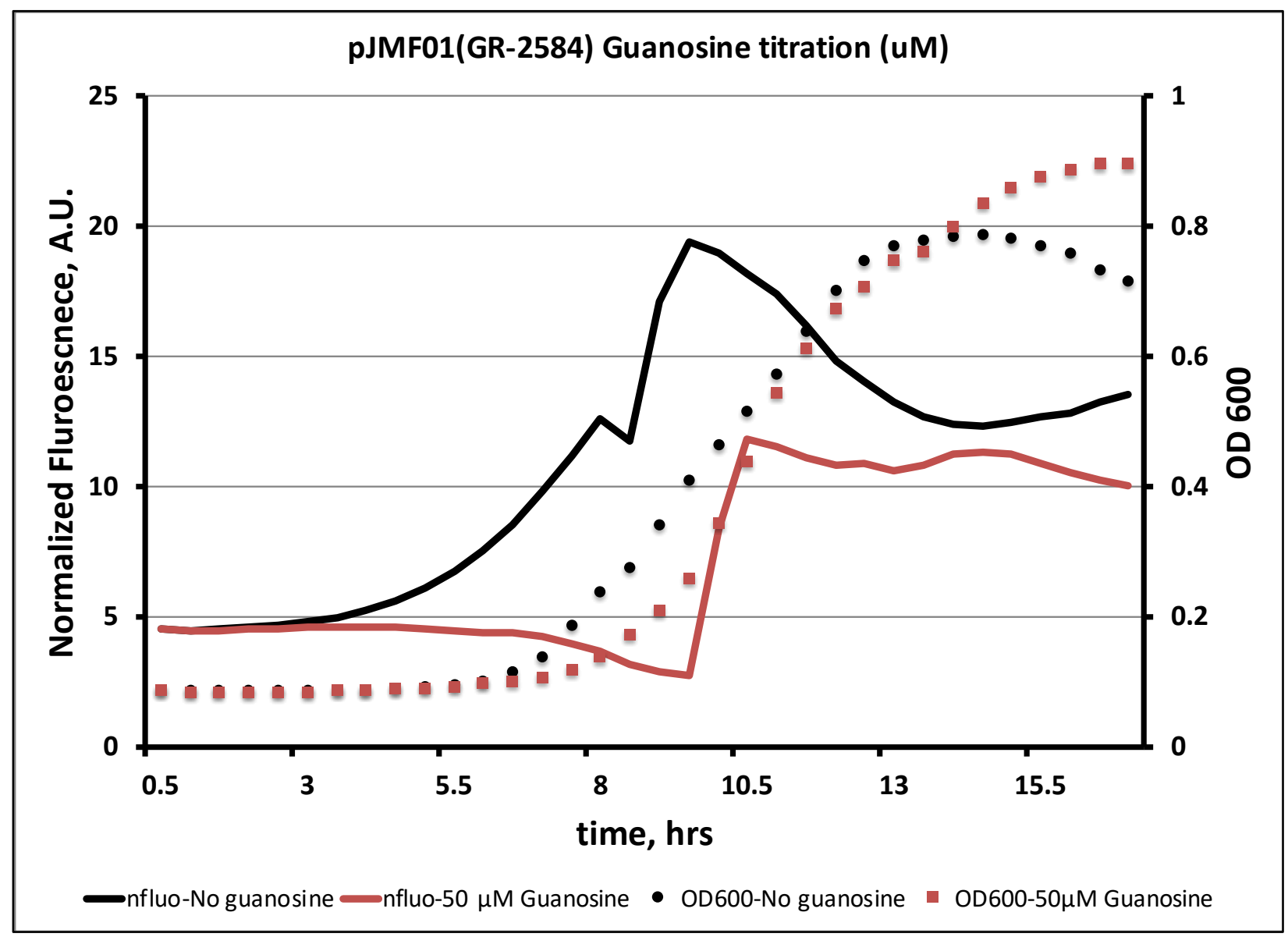

Figure S2. Normalized fluorescence in cell cultures expressing super-folder GFP.

The normalized fluorescence of the cell culture is defined as:

$$
n f l u o=\frac{\text { raw fluorescence of the culture }}{\text { optical density of the culture (OD600) }}
$$

When the normalized fluorescence is plotted with the growth curve, we observed that maximal normalized fluorescence occurs reproducibly at the mid-exponential growth phase, which is consistently the case across cell cultures with different final ligand concentrations. To compare the normalized fluorescence between cultures growing with different ligand concentrations, 
the normalized fluorescence for each culture was averaged between the mid-log points where the OD was between 0.4 and 0.6 .

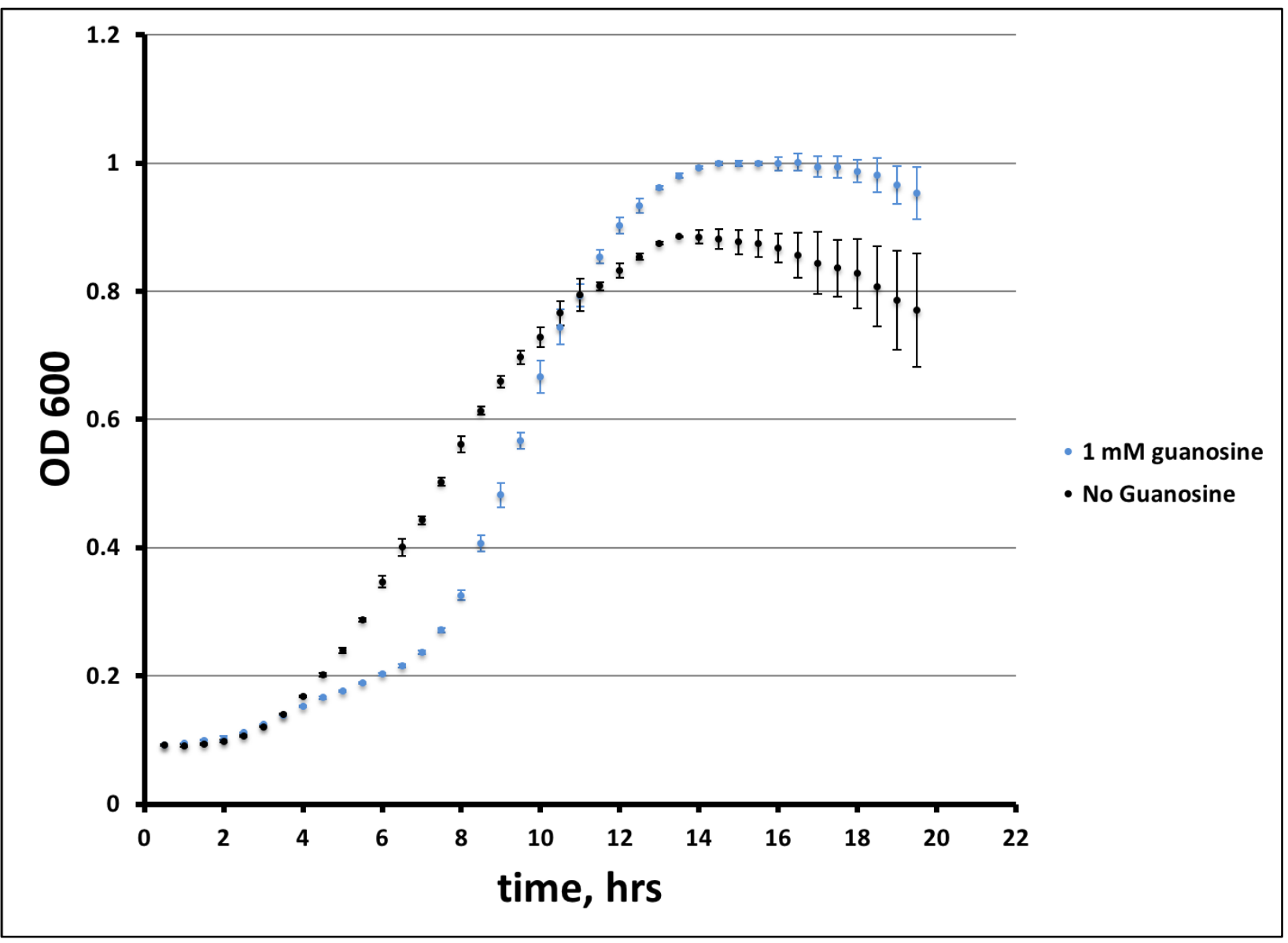

Figure S3. Growth of G. thermoglucosidasius with and without guanosine added to the media.

In this example, the curves represent the growth of the strains carrying plasmid pJMF01, a derivative of the pG1AK plasmid, with and without adding guanosine to the media. The strain expresses the sfGFP gene driven by the pRp1s promoter and was mediated by Tsac 2584 riboswitch. 


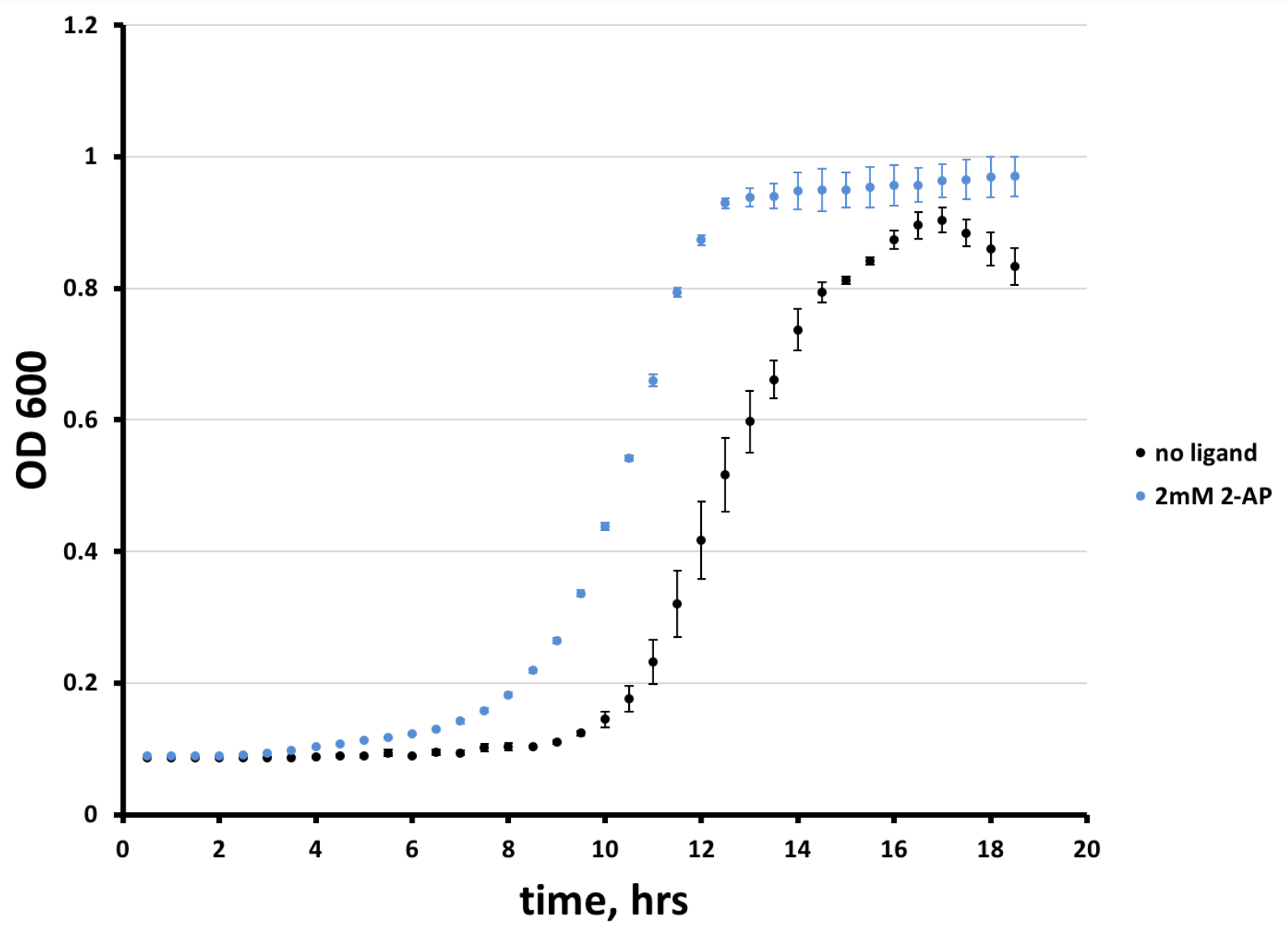

Figure S4. Growth of G. thermoglucosidasius with and without 2-aminopurine added to the media.

In this example, the curves represent the growth of the strains carrying a plasmid that expressed the sfGFP gene under the control of the pbuE $(P 1=10)$ riboswitch and the pRp1s promoter, with and without adding 2-aminopurine to the media. 


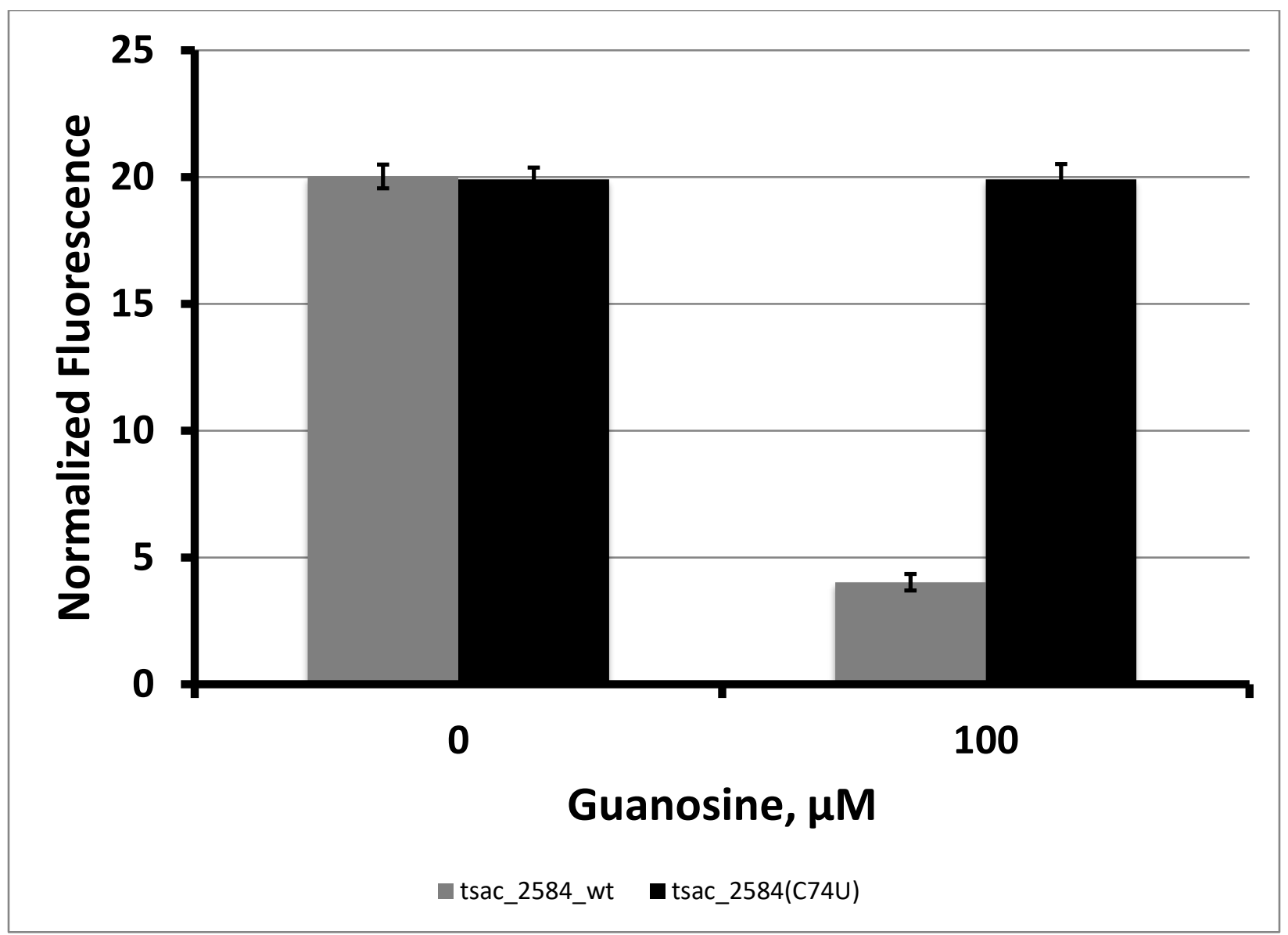

Figure S5. Tsac 2584 non-binding mutant control.

To validate that the regulatory response observed from the $T$. saccharolyticum is indeed due to guanosine-dependent riboswitch activity, we introduced a mutation in the aptamer of the Tsac 2584 riboswitch at the conserved position where guanine interacts with the RNA through a canonical Watson-Creek interaction (cytosine at position 74 to a uridine). In the context of this mutant we no longer observe the regulatory response induced by the wild-type riboswitch when guanosine was added. 


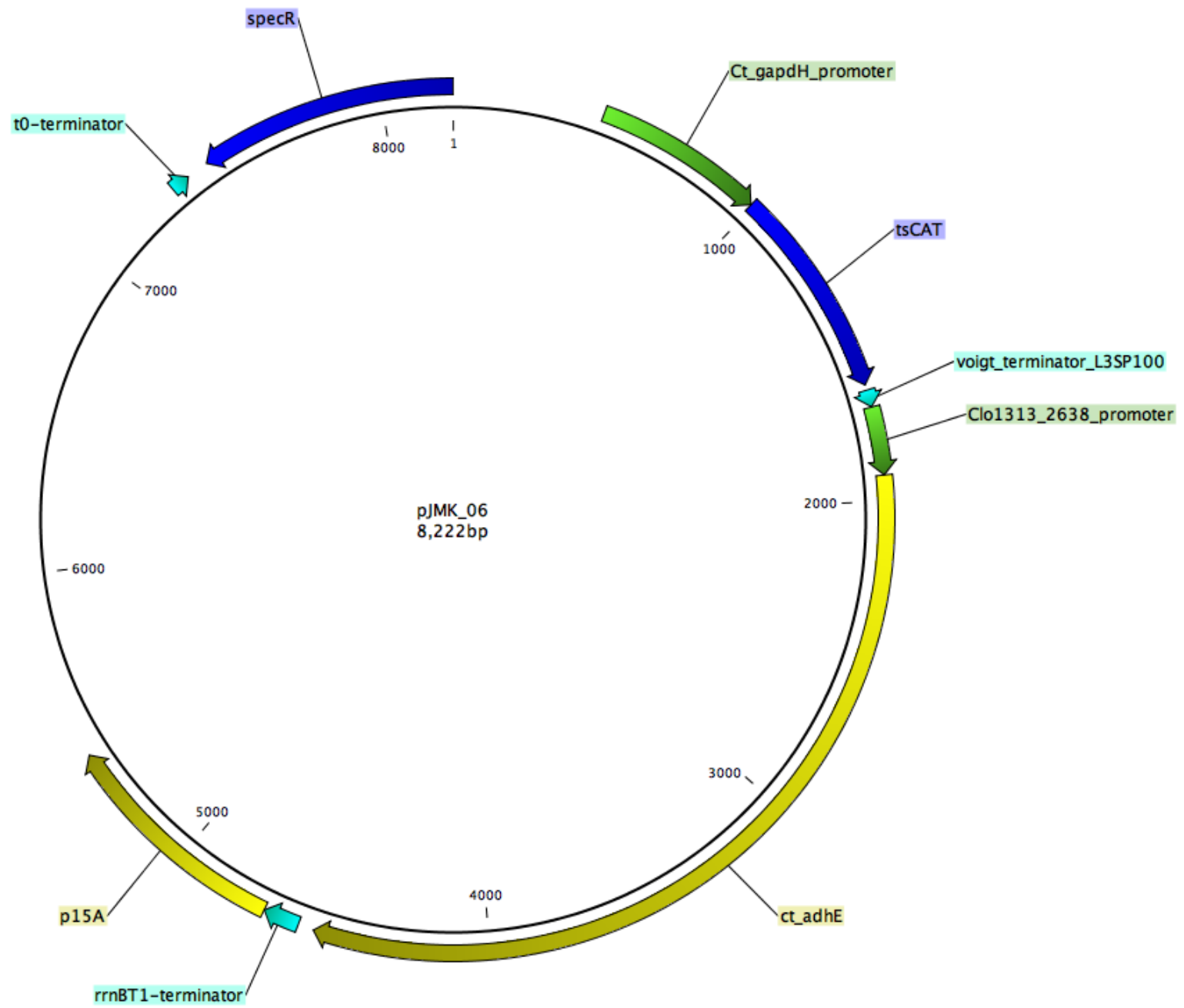

Figure S6. Plasmid Map of pJMK06 plasmid.

This plasmid was designed in house to replicate both in $E$. coli and $C$. thermocellum. A synthetic terminator insulates the expression of the adhE gene from the $C$. thermocellum gapDH promoter that drive the CAT gene from resistance to thiamphenicol. 


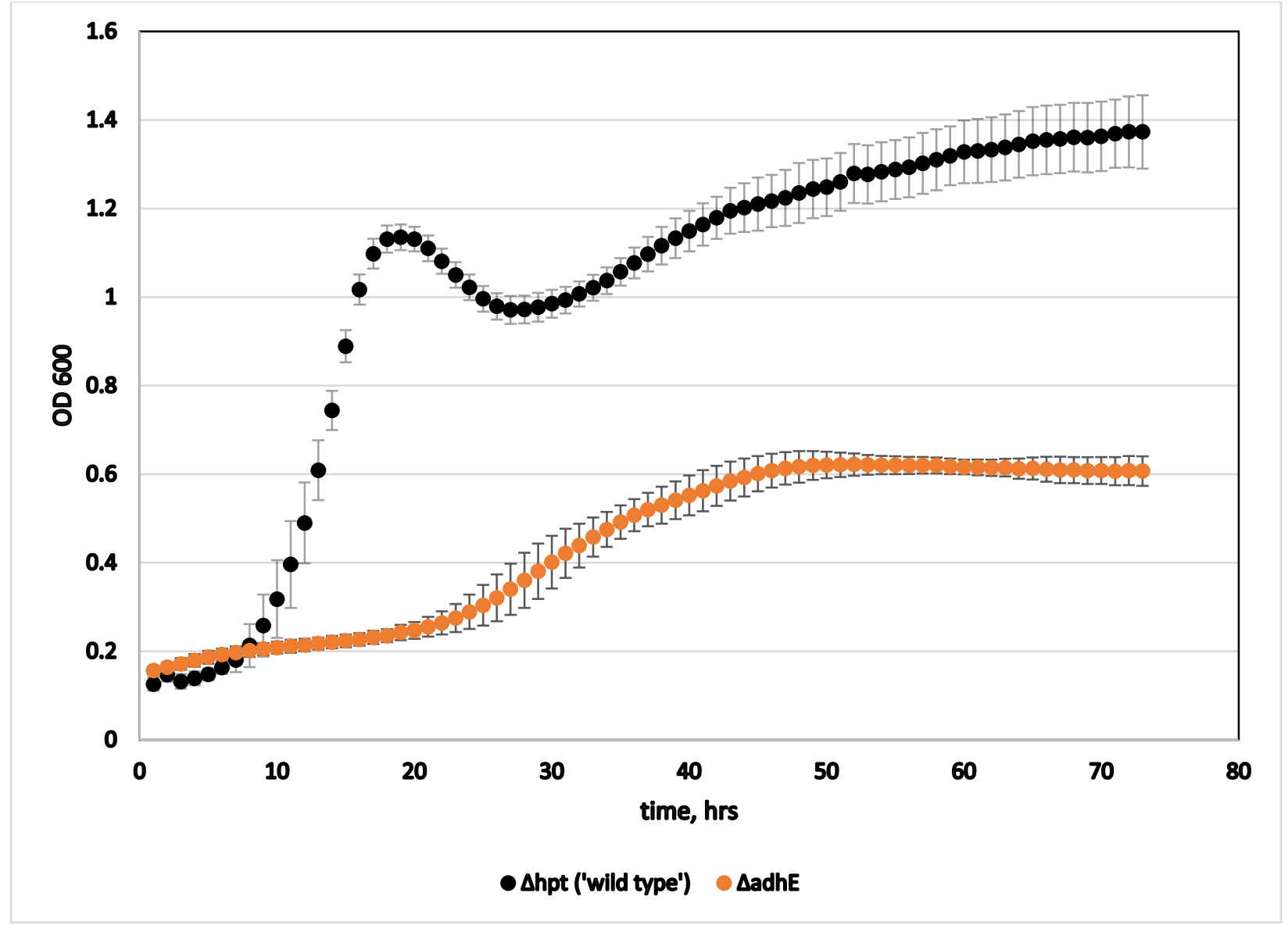

Figure S7. Growth comparison between $\Delta h p t$ ("wild-type") and $\Delta a d h E$.

To enable a genetically tractable strain of Clostridium thermocellum we created a deletion mutant to the hypoxanthine phosphoribosyl transferase (hpt) gene that allows for counter selection. This strain is designated as the "wild-type" in the main text. This is the parental strain used to construct $\triangle a d h E$ deletion that was used in the plasmid complementation experiments. 


\begin{tabular}{|c|c|c|}
\hline $\begin{array}{c}\text { Tsac } 2584 \\
\text { riboswitch } \\
\text { Construct } \\
\text { (G.t) }\end{array}$ & $\begin{array}{l}\text { AACAATCGTTAAAGCGGACGTTTTTGCGCCGCCCGGATTTGCTT } \\
\text { GAAAACTACCCGCTGACAGAAAAGCAAAAACGATGGATCGAA } \\
\text { GAGTGGAAAAAAGAAAACAGTAGCTATTGCGCATGATACAAG } \\
\text { TTTATGCTACTATATTCCTTGTGCAACTTAAGCGATTTGCTTAAG } \\
\text { CGAGGAAAACGATGTTCCGCTGCAATGATGAAAAAGCATTG } \\
\text { ATTGTGTCAGGACAAGTAAATAATAGCTCATATAATTCTGAGAA } \\
\text { TACGGCTCAGAAGTCTCTACCAGACAACCGTAAATTGTCTGACT } \\
\text { ATGAGTGAAAGTGTACCTGAGGGTTCCAGCCTTATTGTCCATGT } \\
\text { GTAGAAAACAGTAAAGGCGTTCGGACCGAGCGGTACAGGCAT } \\
\text { TGTATTGCCACACCTGTGGGATAAAAGCCCGGGAGGATAGGTT } \\
\text { TCACTCTATGTGTTGGAATCTATCATTCCGGGCTTGTTTATTTT } \\
\text { AAAATTCGTTTTATTTCTAGATAAGGAGTGATTCGAATG }\end{array}$ & $\begin{array}{c}\text { Promoter } \\
\text { (G.t -pRp1S) } \\
\text { Riboswitch } \\
\text { RBS } \\
\text { ATG-sfGFP }\end{array}$ \\
\hline $\begin{array}{c}\text { Tsac } 2588 \\
\text { riboswitch } \\
\text { Construct } \\
\text { (G.t) }\end{array}$ & $\begin{array}{l}\text { AACAATCGTTAAAGCGGACGTTTTTGCGCCGCCCGGATTTGCTT } \\
\text { GAAAACTACCCGCTGACAGAAAAGCAAAAACGATGGATCGAA } \\
\text { GAGTGGAAAAAAGAAAACAGTAGCTATTGCGCATGATACAAG } \\
\text { TTTATGCTACTATATTCCTTGTGCAACTTAAGCGATTTGCTTAAG } \\
\text { CGAGGAAAACGATGTTCCGCTGCAATGATGAAAAAGCATTG } \\
\text { ATTGTGTCAGGACAAGTAAATAATAGCTCATATAATTCTGAGAA } \\
\text { TACGGCTCAGAAGTCTCTACCAGACAACCGTAAATTGTCTGACT } \\
\text { ATGAGTGAAAGTGTACCTGAGGGTTCCAGCCTTATTGTCCATGT } \\
\text { GTAGAAAACAGTAAAGGCGTTCGGACCGAGCGGTACAGGCAT } \\
\text { TGTATTGCCACACCTGTGGGATAAAAGCCCGGGAGGATAGGTT } \\
\text { TCACTCTATGTGTTGGAATCTATCATTCCGGGCTTGTTTATTTT } \\
\text { AAAATTCGTTTTATTTCTAGATAAGGAGTGATTCGAATG }\end{array}$ & $\begin{array}{c}\text { Promoter } \\
\text { (G.t -pRp1S) } \\
\text { Riboswitch } \\
\text { RBS } \\
\text { ATG-sfG }\end{array}$ \\
\hline $\begin{array}{c}\text { Tsac } 2584 \\
\text { (non-binding) } \\
\text { riboswitch } \\
\text { Construct } \\
\text { (G.t) }\end{array}$ & $\begin{array}{l}\text { AACAATCGTTAAAGCGGACGTTTTTGCGCCGCCCGGATTTGCTT } \\
\text { GAAAACTACCCGCTGACAGAAAAGCAAAAACGATGGATCGAA } \\
\text { GAGTGGAAAAAAGAAAACAGTAGCTATTGCGCATGATACAAG } \\
\text { TTTATGCTACTATATTCCTTGTGCAACTTAAGCGATTTGCTTAAG } \\
\text { CGAGGAAAACGATGTTCCGCTGCAATGATGAAAAAGCATTGAT } \\
\text { TGTGTCAGGACAAGTAAATAATAGCTCATATAATTCTGAGAATA } \\
\text { CGGCTCAGAAGTCTCTACCAGACAACCGTAAATTGCTGATTAT } \\
\text { GAGTGAAAGTGTACCTGAGGGTTCCAGCCTTATTGTCCATGTGT } \\
\text { AGAAAACAGTAAAGGCGTTCGGACCGAGCGGTACAGGCATTG } \\
\text { TATTGCCACACCTGTGGGATAAAAGCCCGGGAGGATAGGTTTC } \\
\text { ACTCTATGTGTTGGAATCTATCATTCCGGGCTTGTTTATTTTTAA } \\
\text { AATTCGTTTTATTTTCTAGATAAGGAGTGATTCGAATG }\end{array}$ & $\begin{array}{c}\text { Promoter } \\
\text { (G.t-pRp1S) } \\
\text { Riboswitch } \\
\text { C to U } \\
\text { binding } \\
\text { mutant } \\
\text { RBS } \\
\text { ATG-sfG }\end{array}$ \\
\hline
\end{tabular}

\begin{tabular}{|c|l|c|}
\hline & AACAATCGTTAAAGCGGACGTTTTTGCGCCGCCCGGATTTGCTT & Promoter \\
pbue $(w \mathrm{t})$ & GAAAACTACCCGCTGACAGAAAAGCAAAAACGATGGATCGAA & (G.t -pRp1S) \\
riboswitch \\
$\begin{array}{c}\text { Construct } \\
\text { (G.t) }\end{array}$ & GAGTGGAAAAAAGAAAAACAGTAGCTATTGCGCATGATACAAG & Riboswitch \\
TTATGCTACTATATTCCTTGTGCAACTTAAGCGATTTGCTTAAG & RBS \\
\hline
\end{tabular}




\begin{tabular}{|c|c|c|}
\hline & $\begin{array}{l}\text { GGAAACGAATCAATTAAATAGCTATTATCACTTGTATAACCTCA } \\
\text { ATAATATGGTTTGAGGGTGTCTACCAGGAACCGTAAAATCCTG } \\
\text { ATTACAAAATTTGTTTATGACATTTTTTGTAATCAGGATTTTTTTT } \\
\text { ATTTATCAAAACATTTAAGTAAATCTAGATAAGGAGTGATTCGA } \\
\text { ATG }\end{array}$ & \\
\hline $\begin{array}{l}\text { pbue } \\
(\mathrm{P} 1=8) \\
\text { riboswitch } \\
\text { Construct } \\
\text { (G.t) }\end{array}$ & $\begin{array}{l}\text { AACAATCGTTAAAGCGGACGTTTTTGCGCCGCCCGGATTTGCTT } \\
\text { GAAAACTACCCGCTGACAGAAAAGCAAAAACGATGGATCGAA } \\
\text { GAGTGGAAAAAAGAAAAACAGTAGCTATTGCGCATGATACAAG } \\
\text { TTTATGCTACTATATTCCTTGTGCAACTTAAGCGATTTGCTTAAG } \\
\text { CGAGGAAAACGATGTTCCGCTGCAATGATGAAAAAGCATTG } \\
\text { GGAAACGAATCAATTAAATAGCTATTATATTTTGTATAACCTCA } \\
\text { ATAATATGGTTTGAGGGTGTCTACCAGGAACCGTAAAATCCTG } \\
\text { ATTACAAAATTTGTTTATGACATTTTTTTAATCAGGATTTTTTT } \\
\text { ATTTATCAAAACATTTAAGTAAATCTAGATAAGGAGTGATTCGA } \\
\text { ATG }\end{array}$ & $\begin{array}{c}\text { Promoter } \\
\text { (G.t -pRp1S) } \\
\text { Riboswitch } \\
\text { mutation } \\
\text { RBS } \\
\text { ATG-sfG }\end{array}$ \\
\hline $\begin{array}{l}\text { pbue } \\
(\mathrm{P} 1=10) \\
\text { riboswitch } \\
\text { Construct } \\
\text { (G.t) }\end{array}$ & $\begin{array}{l}\text { AACAATCGTTAAAGCGGACGTTTTTGCGCCGCCCGGATTTGCTT } \\
\text { GAAAACTACCCGCTGACAGAAAAGCAAAAACGATGGATCGAA } \\
\text { GAGTGGAAAAAAGAAAAACAGTAGCTATTGCGCATGATACAAG } \\
\text { TTTATGCTACTATATTCCTTGTGCAACTTAAGCGATTTGCTTAAG } \\
\text { CGAGGAAAACGATGTTCCGCTGCAATGATGAAAAAGCATTG } \\
\text { GGAAACGAATCAATTAAATAGCTATTAAATTTTGTATAACCTCA } \\
\text { ATAATATGGTTTGAGGGTGTCTACCAGGAACCGTAAAATCCTG } \\
\text { ATTACAAAATTTGTTATGACATTTTTGTAATCAGGATTTTTTT } \\
\text { ATTTATCAAAACATTTAAGTAAATCTAGATAAGGAGTGATTCGA } \\
\text { ATG }\end{array}$ & $\begin{array}{c}\text { Promoter } \\
\text { (G.t -pRp1S) } \\
\text { Riboswitch } \\
\text { mutation } \\
\text { RBS } \\
\text { ATG-sfG }\end{array}$ \\
\hline
\end{tabular}

\begin{tabular}{|c|c|c|}
\hline $\begin{array}{c}\text { adhe } \\
\text { constitutive } \\
\text { expression } \\
\text { (C.t) }\end{array}$ & $\begin{array}{l}\text { GATAAACAAAGGACGGTTCAGGGCTTCTGCTCATCCTACTCT } \\
\text { GCATTGTAAAAAGGTAGGATGAATTTTTATTTTTAATCTTATT } \\
\text { GAAAAAAATTTTTGAAAATCGGTTTTATTAAAAAAAAGTGG } \\
\text { GTATATTTATAATAGTCAATTGATTGGTTAAAAAAATTTAAAT } \\
\text { AAGCAAACAGAATAATAACAAAAGTAAGGAGGAATTTGTTA } \\
\text { TG }\end{array}$ & $\begin{array}{c}\text { Promoter } \\
\text { (Clo1515_2365) } \\
\text { ATG-adhE }\end{array}$ \\
\hline $\begin{array}{c}\text { Adhe } \\
\text { pbuE (wt) } \\
\text { (C.t.) }\end{array}$ & $\begin{array}{l}\text { GATAAACAAAGGACGGTTCAGGGCTTCTGCTCATCCTACTCT } \\
\text { GCATTGTAAAAAGGTAGGATGAATTTTTATTTTAATCTTATT } \\
\text { GAAAAAAATTTTGAAAATCGGTTTTATTAAAAAAAATGG } \\
\text { GTATATTTATAATAGTCAATTGATTGGTTAAAAAAATTTAAAT } \\
\text { AAGCAAACAGAATAATAAGGAAACGAATCAATTAAATAGCT } \\
\text { ATTATCACTTGTATAACCTCAATAATATGGTTTGAGGGTGTCT } \\
\text { ACCAGGAACCGTAAAATCCTGATTACAAAATTTGTTTATGAC } \\
\text { ATTTTTTGTAATCAGGATTTTTTTATTTATCAAAACATTTAAG } \\
\text { TAAAAAAAGTAAGGAGGAATTTGTATG }\end{array}$ & $\begin{array}{c}\text { Promoter } \\
\text { (Clo1515_2365) } \\
\text { Riboswitch } \\
\text { ATG-adhE }\end{array}$ \\
\hline pbue & $\begin{array}{l}\text { GATAAACAAAGGACGGTTCAGGGCTTCTGCTCATCCTACTCT } \\
\text { GCATTGTAAAAAGGTAGGATGAATTTTTATTTTTAATCTTATT }\end{array}$ & $\begin{array}{l}\text { Promoter } \\
\text { (G.t-pRp1S) }\end{array}$ \\
\hline
\end{tabular}




\begin{tabular}{|c|c|c|}
\hline $\begin{array}{c}(\mathrm{P} 1=8) \\
\text { riboswitch } \\
\text { Construct } \\
\text { (C.t) }\end{array}$ & $\begin{array}{l}\text { GAAAAAAATTTTTGAAAATCGGTTTTATTAAAAAAAAGTGG } \\
\text { GTATATTTATAATAGTCAATTGATTGGTTAAAAAAATTTAAAT } \\
\text { AAGCAAACAGAATAATAAGGAAACGAATCAATTAAATAGCT } \\
\text { ATTATATTTTGTATAACCTCAATAATATGGTTTGAGGGTGTCT } \\
\text { ACCAGGAACCGTAAAATCCTGATTACAAAATTTGTTTATGAC } \\
\text { ATTTTTTGTAATCAGGATTTTTTTATTTATCAAAACATTTAAG } \\
\text { TAAAAAAAGTAAGGAGGAATTTGTTATG }\end{array}$ & $\begin{array}{c}\text { Riboswitch } \\
\text { mutation } \\
\text { RBS } \\
\text { ATG-sfG }\end{array}$ \\
\hline
\end{tabular}

Supporting Table S1. Riboswitch construct sequences in this study. 


\begin{tabular}{|c|c|c|c|c|c|c|}
\hline \multicolumn{7}{|c|}{ Plasmids developed for this study } \\
\hline $\begin{array}{c}\text { Plasmid } \\
\text { name }\end{array}$ & Promoter & Riboswitch & Reporter & $\begin{array}{l}\text { Mode of } \\
\text { regulation }\end{array}$ & Ligand & $\begin{array}{c}\text { Host } \\
\text { for } \\
\text { assay }\end{array}$ \\
\hline pJMF01 & pRp1S & Tsac 2584 & sfGFP & "off" & guanosine & G.t. \\
\hline pJMF02 & pRp1S & Tsac 2584 & sfGFP & *mutant & guanosine & G.t. \\
\hline pJMF03 & pRp1S & Tsac 2588 & sfGFP & "off" & guanosine & G.t. \\
\hline pJMF10 & pRp1S & pbuE-wt & sfGFP & "on" & 2-AP & G.t. \\
\hline pJMF11 & pRp1S & pbuE-P1 = 8bp & sfGFP & "on" & 2-AP & G.t. \\
\hline pJMF12 & pRp1S & pbuE-P1 = 10bp & sfGFP & "on" & $2-A P$ & G.t. \\
\hline pJMK06 & Clo1313_2638 & No riboswitch & C.t. adhE & N.A. & 2-AP & C.t. \\
\hline pJMK09 & Clo1313_2638 & pbuE-wt & C.t. adhE & "on" & 2-AP & C.t. \\
\hline pJMK11 & Clo1313_2638 & pbuE-P1 = 8bp & C.t. adhE & "on" & 2-AP & C.t. \\
\hline pJMK12 & Clo1313_2638 & pbuE-P1 = 10bp & C.t. adhE & "on" & $2-A P$ & C.t. \\
\hline
\end{tabular}

Table S2. Plasmids developed for this study. 


\begin{tabular}{|c|c|c|c|c|}
\hline construct & $\begin{array}{c}\text { Lactic acid, } \\
\mathrm{mM}\end{array}$ & $\begin{array}{c}\text { Acetic acid, } \\
\mathrm{mM}\end{array}$ & $\begin{array}{c}\text { Ethanol, } \\
\mathrm{mM}\end{array}$ & $\begin{array}{c}\text { Total products, } \\
\mathrm{mM}\end{array}$ \\
\hline pbuE wt & $11.87 \pm 1.22$ & $7.09 \pm 0.33$ & $1.73 \pm 0.16$ & $20.68 \pm 1.62$ \\
\hline pbuE wt + 2AP & $\mathbf{1 5 . 7 7} \pm \mathbf{0 . 5 1}$ & $\mathbf{9 . 3 5} \pm \mathbf{0 . 0 7}$ & $\mathbf{3 . 7 8} \pm \mathbf{0 . 0 6}$ & $\mathbf{2 8 . 9 0 \pm 0 . 6 4}$ \\
\hline pbuE (P1=8) & $14.08 \pm 1.66$ & $7.62 \pm 0.68$ & $2.38 \pm 0.50$ & $24.09 \pm 2.72$ \\
\hline pbuE (P1=8) + 2AP & $\mathbf{1 0 . 6 4} \pm \mathbf{0 . 0 2}$ & $\mathbf{1 1 . 1 4} \pm \mathbf{0 . 5 7}$ & $\mathbf{1 5 . 6 1} \pm \mathbf{5 . 2 9}$ & $\mathbf{3 7 . 3 9} \pm \mathbf{5 . 8 1}$ \\
\hline pbuE (P1=10) & $14.92 \pm 0.78$ & $7.50 \pm 1.65$ & $6.38 \pm 1.41$ & $28.80 \pm 2.34$ \\
\hline pbuE (P1=10) + 2AP & $\mathbf{8 . 8 7} \pm \mathbf{0 . 4 4}$ & $\mathbf{1 0 . 1 4} \pm \mathbf{0 . 1 5}$ & $\mathbf{1 7 . 3 1 \pm 0 . 4 4}$ & $\mathbf{3 6 . 3 2} \pm \mathbf{1 . 0 0}$ \\
\hline no riboswitch & $2.18 \pm 0.59$ & $4.60 \pm 1.34$ & $8.20 \pm 3.28$ & $14.98 \pm 5.21$ \\
\hline no riboswitch + 2AP & $\mathbf{2 . 1 3} \pm \mathbf{0 . 3 5}$ & $\mathbf{4 . 3 8 \pm \mathbf { 0 . 9 5 }}$ & $\mathbf{8 . 0 2} \pm \mathbf{2 . 4 0}$ & $\mathbf{1 4 . 5 3 \pm 3 . 7 1}$ \\
\hline
\end{tabular}

\begin{tabular}{|c|c|c|c|}
\hline construct & Lactic acid \% & Acetic acid \% & Ethanol \% \\
\hline pbuE wt & $57 \pm 2$ & $34 \pm 2$ & $8 \pm 1$ \\
\hline pbuE wt + 2AP & $\mathbf{5 5} \pm \mathbf{1}$ & $\mathbf{3 2} \pm \mathbf{1}$ & $\mathbf{1 3} \pm \mathbf{1}$ \\
\hline pbuE (P1=8) & $58 \pm 1$ & $32 \pm 1$ & $10 \pm 1$ \\
\hline pbuE (P1=8) + 2AP & $\mathbf{2 9} \pm \mathbf{4}$ & $\mathbf{3 0} \pm \mathbf{3}$ & $\mathbf{4 1} \pm \mathbf{7}$ \\
\hline pbuE (P1=10) & $52 \pm 6$ & $26 \pm 3$ & $22 \pm 3$ \\
\hline pbuE (P1=10) + 2AP & $\mathbf{2 4} \pm \mathbf{1}$ & $\mathbf{2 8} \pm \mathbf{1}$ & $\mathbf{4 8} \pm \mathbf{1}$ \\
\hline no riboswitch & $15 \pm 1$ & $31 \pm 2$ & $54 \pm 3$ \\
\hline no riboswitch + 2AP & $\mathbf{1 5} \pm \mathbf{1}$ & $\mathbf{3 0} \pm \mathbf{1}$ & $\mathbf{5 5} \pm \mathbf{3}$ \\
\hline
\end{tabular}

Table S3. Quantification of $C$. thermocellum fermentation products.

The millimolar amounts of the fermentation products were quantified by HPLC. The numbers represent the average values of three independent experiments. 


\section{Supporting Methods}

Media Recipes

Defined Glucose Media (DG)- 1000mL

\begin{tabular}{|c|c|}
\hline Minimal Salts (below) & $20 \mathrm{~mL}$ \\
\hline $10 \% \mathrm{KOH}(\mathrm{w} / \mathrm{v})$ & $0.68 \mathrm{~mL}$ \\
\hline water & $830 \mathrm{~mL}$ \\
\hline \multicolumn{2}{|c|}{ After autoclave add the following: } \\
\hline 1.05 M nitrilotriacetic acid & $1 \mathrm{~mL}$ \\
\hline $0.59 \mathrm{MgSO}_{4} \cdot 7 \mathrm{H}_{2} \mathrm{O}$ & $1 \mathrm{~mL}$ \\
\hline $0.91 \mathrm{M} \mathrm{CaCl}_{2} \cdot 2 \mathrm{H}_{2} \mathrm{O}$ & $1 \mathrm{~mL}$ \\
\hline $0.04 \mathrm{M} \mathrm{FeSO}_{4} \cdot 7 \mathrm{H}_{2} \mathrm{O}$ & $1 \mathrm{~mL}$ \\
\hline $50 \%(\mathrm{v} / \mathrm{v})$ Glycerol & $8 \mathrm{~mL}$ \\
\hline Trace Metal A6 (below) & $1 \mathrm{~mL}$ \\
\hline $10 \%(w / v)$ Casaminoacids & $10 \mathrm{~mL}$ \\
\hline $40 \mathrm{~g} / \mathrm{L}$ Sodium Pyruvate & $100 \mathrm{~mL}$ \\
\hline $20 \%$ Glucose & $25 \mathrm{~mL}$ \\
\hline Vitamix Mix 500X (below) & $2 \mathrm{~mL}$ \\
\hline
\end{tabular}

\begin{tabular}{|c|c|}
\hline \multicolumn{2}{|c|}{ Vitamin mix 500X-25mL } \\
\hline Component & $\mathbf{m g} / \mathbf{2 5} \mathbf{m L}$ \\
\hline pyridoxamine HCL & 50 \\
\hline Biotin & 5 \\
\hline p-Aminobenzoic acid & 10 \\
\hline Vitamin B12 & 5 \\
\hline
\end{tabular}

\begin{tabular}{|l|l|}
\hline \multicolumn{2}{|c|}{ Minimal Salts } \\
\hline Component & Grams/Litre \\
\hline $\mathrm{K}_{2} \mathrm{HPO}_{4}$ & 26.1 \\
\hline $\mathrm{KH}_{2} \mathrm{PO}_{4}$ & 11.3 \\
\hline $\mathrm{NH}_{4} \mathrm{NO} 3$ & 25 \\
\hline
\end{tabular}

\begin{tabular}{|c|c|}
\hline \multicolumn{2}{|c|}{ Trace Metal A5 } \\
\hline Component & $\mathrm{mg} / \mathrm{L}$ \\
\hline $\mathrm{H}_{3} \mathrm{BO}_{3}$ & 2860 \\
\hline $\mathrm{MnCl}_{2}$ & 1810 \\
\hline $\mathrm{ZnMoO}_{4}$ & 390 \\
\hline $\mathrm{CuSO}_{4} \cdot 7 \mathrm{H}_{2} \mathrm{O}$ & 79 \\
\hline $\mathrm{Co}\left(\mathrm{NO}_{3}\right)_{2} \cdot 6 \mathrm{H}_{2} \mathrm{O}$ & 49 \\
\hline $\mathrm{ZnSO}_{4} \cdot 7 \mathrm{H}_{2} \mathrm{O}$ & 222 \\
\hline
\end{tabular}


TGP media recipe $1000 \mathrm{~mL}$ \{Cripps:2009fx $\}$

$17 \mathrm{~g}$ - Tryptone

$3 \mathrm{~g}$ - Soy Peptone

$2.5 \mathrm{~g}-\mathrm{KH}_{2} \mathrm{PO}_{4}$

$5 \mathrm{~g}-\mathrm{NaCl}$

pH 7.3 pre-autoclaving

After autoclave add:

$40 \mathrm{~g} / \mathrm{L}$ sodium pyruvate $-4.0 \mathrm{~mL}$

$50 \%$ glycerol $-8.0 \mathrm{~mL}$

\section{C. thermocellum growth media and strain development}

The C.t. culturing media, CTFUD, was made following a protocol published previously \{Olson:2012gi\} The adhE gene was targeted deleted in an in-house and unpublished host strain in which the entire non-coding region containing the promoter of the clo1313_2638 gene was inserted immediately upstream of the gene encoding for the evolving hydrogenase catalytic subunit (clo1313_1791) in the genome. The altered hydrogenase gene expression facilitated the targeted deletion of $a d h E$ gene in C.t. The marke-rless adhE gene deletion was done by double homologous recombination following the selection and counter-selection methods described previously \{Olson:2012gi\}.

Identification of riboswitch sequences in thermophilic bacteria

The INFERNAL software was used in conjunction with shell scripts to search for 25 riboswitch covariance models against 73 representative thermophilic bacterial genomic sequences that were annotated as complete genomes in the NCBI nucleotide database and most of which were included in the well curated NCBI RefSeq database. The genomes were downloaded from the NCBI web-portal as a multi-fasta file and split into individual fasta files for each of the genomes using a Biopython script. Awk and other shell scripts were developed in-house to analyze the output from the INFERNAL software. This analysis indicated which of the 25 types of riboswitches in the riboswitch covariance model database were present in the chosen representative thermophilic bacteria and the relative frequencies/percentages of the different types of riboswitches in these bacteria.

The complete alignment of the RFAM Purine Riboswitch family (RF00167) was graciously provided by RFAM researchers at EBI (European Bioinformatics Institute). In-house shell scripts were used to process the complete alignment to yield a tabulated and annotated version of the complete purine riboswitch alignment. The tabulated and annotated complete purine riboswitch alignment was next processed using an in-house Python script to identify the 
frequency of the four possible ribonucleotides at three functionally key positions $(22,51$ and 74) from an aptamer region in the purine riboswitches ${ }^{1}$. This investigation revealed that the most functionally significant conserved position (74) in the purine riboswitches was always devoid of the Uracil ribonucleotide in thermophilic organisms. This Uracil ribonucleotide is a signature of adenine riboswitches which in turn indicates that adenine riboswitches are absent in thermophiles.

\section{Quantification of fermentation products}

One milliliter of the culture was centrifuged, and the supernatant was filtered through a 0.22 $\mu \mathrm{M}$ membrane. Fermentation products including ethanol, acetic acid, and lactic acid were measured by HPLC (1200 series; Agilent Technologies, Santa Clara, CA) with a mobile phase of 4 $\mathrm{mM}$ sulfuric acid (flow rate of $0.6 \mathrm{ml} / \mathrm{min}$ ) using an Aminex HPX-87H column with a Micro Guard Cation H Cartridge. The column temperature was set to $55^{\circ} \mathrm{C}$.

\section{Supplemental References}

(1)Weinberg, Z., Nelson, J. W., Lünse, C. E., Sherlock, M. E., and Breaker, R. R. (2017) Bioinformatic analysis of riboswitch structures uncovers variant classes with altered ligand specificity. Proc. Natl. Acad. Sci. U.S.A. 114, E2077-E2085. 